\title{
Electric Field-Induced Point Defect Redistribution in $\mathrm{TiO}_{2}$
}

\author{
Ali Moballegh and Elizabeth C. Dickey
}

Department of Materials Science and Engineering, North Carolina State University, Raleigh, NC, USA

The resistance switching behavior of transition metal oxides has attracted increasing interest for potential use in nonvolatile memory devices [1]. The switching behavior is believed to result from the migration of ionic carriers during an "electroforming" process, in which charged point defects diffuse in response to a high electric field stress. In $\mathrm{TiO}_{2}$, this involves the migration of oxygen vacancies and titanium interstitials. The redistribution of ionic carriers leads to heterogeneity in the stoichiometry of $\mathrm{TiO}_{2}$, which can be in the form of nonstoichiometric filaments oriented along the applied field direction. Since the electronic conductivity of $\mathrm{TiO}_{2}$ is very sensitive to stoichiometry, this redistribution can have a profound effect on the electrical properties. The present work aims to understand the redistribution process at the mesoscopic length scale as a function of the initial defect chemistry state and the electrode boundary conditions. In addition, experiments are performed as a function of crystallographic orientation since the self-diffusion coefficients of oxygen vacancies and titanium interstitials are known to be highly anisotropic in rutile $[2,3]$.

The initial-state point defect concentrations are controlled by equilibrating single crystals at specific oxygen partial pressures and temperatures [4]. Metallic electrodes are deposited by a DC sputtering system with a thickness of approximately $300 \mathrm{~nm}$. Platinum or gold contacts are used in order to establish Schottky contacts. The samples are then subjected to electric field strengths as high as 200 $\mathrm{V} / \mathrm{cm}$; while the leakage current is continuously monitored. After electrical degradation, the samples are interrogated by a combination of electron microscopy techniques that provide insight into the point defect redistribution.

Figure 1a shows cathodoluminescence (CL) spectra from two distinct regions in a field-degraded $\mathrm{TiO}_{2}$ crystal. As shown in the inset optical image, dark filaments appear to orient along the applied field direction (Fig. 1b). The CL spectrum taken from a dark filament exhibits a noticeable increase in the magnitude of the emission spectra at $\sim 780 \mathrm{~nm}$; this peak has previously been attributed to titanium interstitials [5,6]. Figure 1c shows a composite CL image acquired using blue and red filters, as indicated in the spectra, showing the correlation of the point defects within the dark filament regions. The CL technique proves to be very useful for quantifying the spatial distribution of the relevant defects in the microstructure.

To further understand local microchemistry, but at the nanometer to atomic length scale, we also prepare site-selective TEM samples by focused ion beam (FIB). Figure 2a shows the extraction of a sample near the cathode region of a field-degraded sample. Electron energy loss spectroscopy (EELS) is performed in a monochromated FEI G2 Titan to determine the local stoichiometry and cation valence at the electrode region (Fig. 2b) [7]. 


\section{References:}

[1] R. Waser et al, Advanced Materials 21 (2009), 2632-2663.

[2] H. Iddir et al, Physical Review B 75 (2007), 073203.

[3] D. K. Lee, H. I. Yoo, Solid State Ionics 177 (2006), 1-9.

[4] M. Nowotny et al, J. Phys. Chem. B, 110 (2006), 16270-16282.

[5] I. Fernandez et al, Sci. Technol. 20 (2005), 239-243.

[6] R. Plugaru et al, J. Phys.: Condens. Matter 16 (2004) S261-S268.

[7] The authors acknowledge funding from the National Science Foundation, Grant Number DMR 1132058.
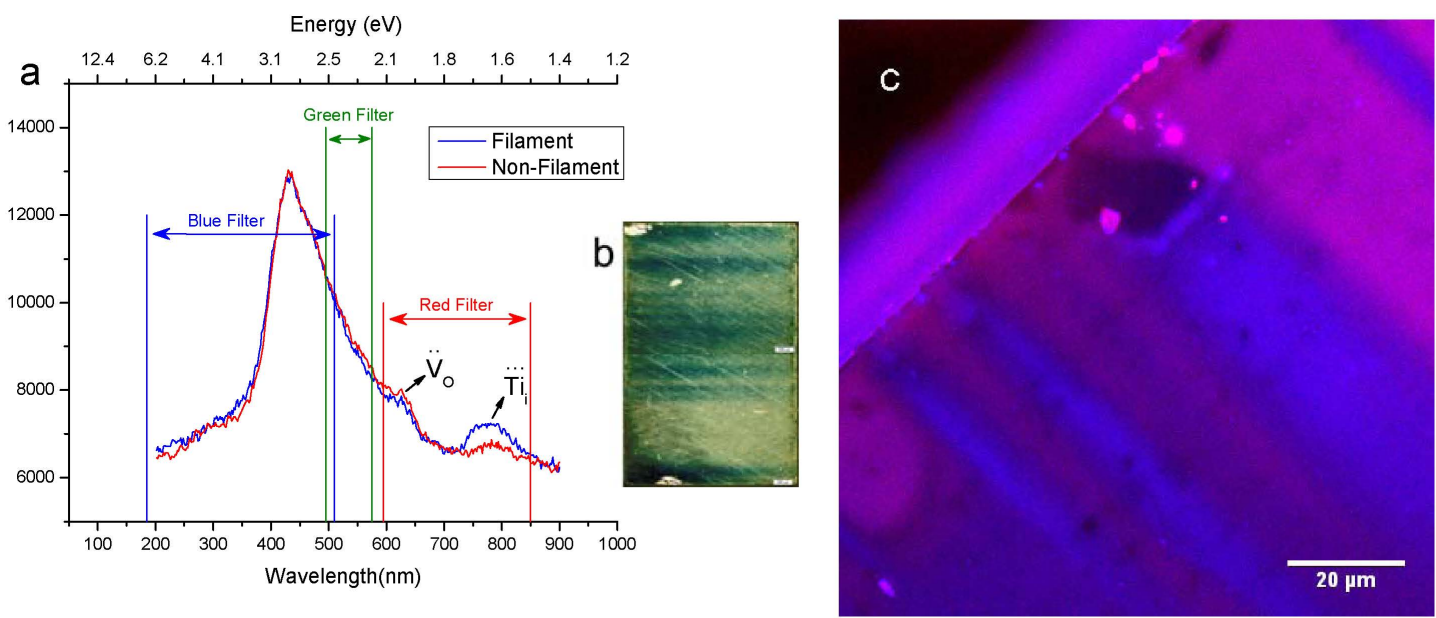

Figure 1. (a) Cathodoluminescence spectra of a reduced single-crystal $\mathrm{TiO}_{2}$ sample. Red curve is taken from the bulk and blue curve is from a filament region. The spectra were taken at $298 \mathrm{~K}$ with an electronbeam energy of $20 \mathrm{keV}$. (b) An optical image of a field-degraded $\mathrm{TiO}_{2}$ crystal. (c) A composite of CL images taken from the $\mathrm{TiO}_{2}$. One is taken by using UV-blue filter (blue color) and another image is taken by red filter (red color). The images were taken at 750x with an electron-beam energy of $20 \mathrm{keV}$.
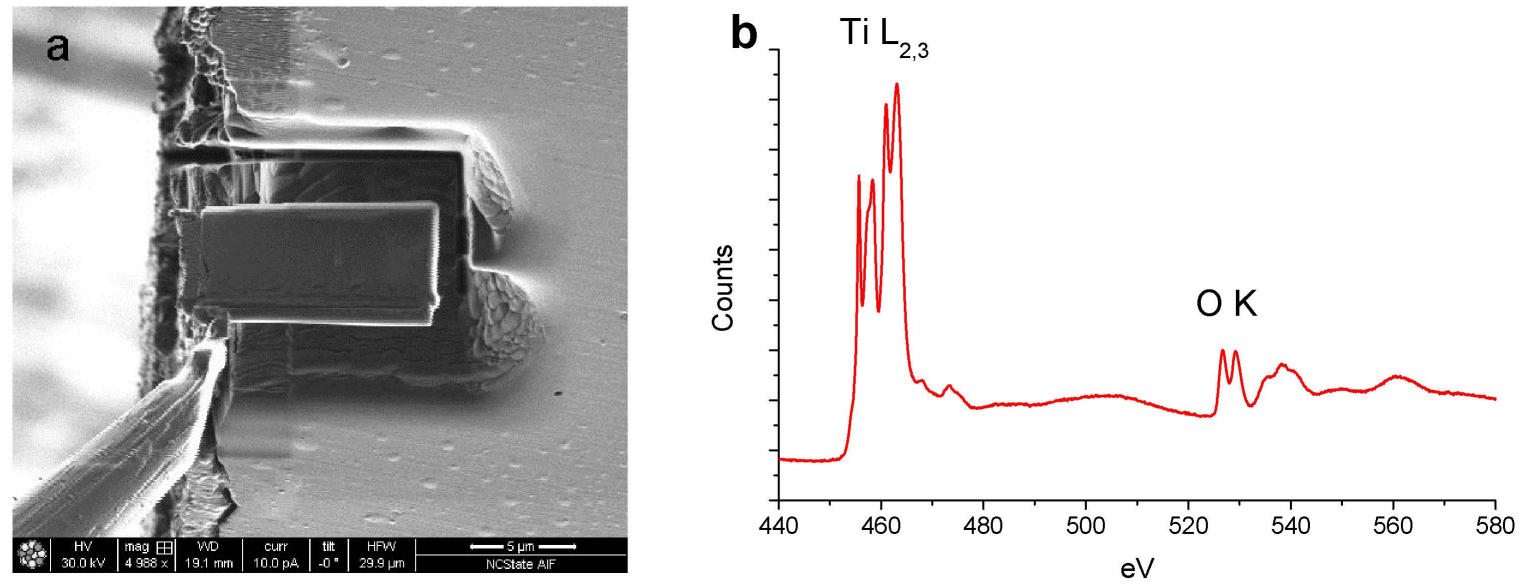

Figure 2. (a) TEM sample preparation by FIB from the near-electrode regions. (b) The EEL spectrum of the reduced single-crystal $\mathrm{TiO}_{2}$ after electric field degradation from a region adjacent to the cathode. 